\title{
Experimental Research on English Reading Behavior Based on E-Prime System
}

\section{Feng Zhu}

School of Foreign Languages, Taizhou University, Taizhou 225300, Jiangsu, China Email: zhufeng6388@163.com

\begin{abstract}
Guided by the framework of structural construction and based on the E-Prime experimental generation system, the reading time method and detection technology are used to investigate the differences of reading comprehension between conditional adverbial clauses which presented by two ways - preposition and postposition among native Chinese college students. It is found that there are significant differences in reading time and understanding between the preposition and postposition of conditional adverbial clauses in English reading. The results show that both at the sentence level and at the text level, the postposition structure is more difficult to characterize than the preposition structure, which affects the reading speed and the accuracy of understanding. The study aims to improve English reading teaching.

Keywords: English reading, sentence structure, structural construction framework, reading time method, detection techniques
\end{abstract}

\section{Introduction}

Before 1970 s, reading research was mainly based on off-line memory measurement. ${ }^{[1]}$ In recent years, the academic circles began to study the nature of reading from the perspective of cognitive psychology by using the on-line method. ${ }^{[2]}$ Scholars at home and abroad have applied structural construction theory to L2 reading research, such as Wang Suiping, Mo Lei (2001), Wu Shiyu, Wang Tongshun (2006), Elizabeth (2006), Wu Shiyu, Hu Wenjie (2007), etc. ${ }^{[1-4]}$ On the basis of previous studies, this paper attempts to use the Structure Building Framework of cognitive psychology to study the influence of preposition and postposition of conditional adverbial clauses on reading comprehension in L2 reading by real-time method. While examining language factors, it mainly studies the nature of differences in reading comprehension between preposition and postposition of conditional clauses from the perspective of cognitive psychology.

\section{Theoretical framework}

Modern psycholinguist (Kintsch W, 1988) think that reading is a complex cognitive process. ${ }^{[5]}$ In 1990 , Morton Ann Gernsbacher, a professor of psychology and psycholinguistics at Madison University, Wisconsin, put forward the theory of structural construction, which holds that understanding consists of three main processes: laying a foundation, mapping and shifting. ${ }^{[6]}$ Specifically, it is understood that when the current information and the previous information are coherent (laying a foundation), it is easier for readers to activate similar memory chunks, so the current information is easier to map a new structure. On the contrary, it takes more time to transfer, and Elizabeth's research (2006) also verified this conclusion.

To form a coherent psychological representation, the ability to logically relations in perceptual texts is an important factor. (Elizabeth, 2006) ${ }^{[3]}$ What is a logical relationship? Winter (1975) once gave the definition as: "Two semantic units related to each other, and one of which complements the other". ${ }^{[7]}$ In 1983, Fahnestock put forward that "depending on whether the second unit meets readers' expectations". ${ }^{[8]}$ If it meets, it represents normal expectations, and understanding is continuous; otherwise, it will lead to accidents, and understanding cannot be continuous. Then, how to understand the continuity and discontinuity of the preposition and postposition of conditional clauses in this study? Zhang Zhenbang (2003) expounded the influence of different positions of adverbial clauses on understanding from the perspective of semantic relations, and held that the pre-structure (The adverbial clause comes first, and the main clause comes last) can best reflect the semantic master-slave relationship (The center of gravity is behind). Post-structure (The main sentence comes first, and the clauses come last) often causes the change of semantic center of gravity (The center of gravity is ahead). ${ }^{[9]}$ At the same time, the first language experience also influences the syntactic processing of the second language, which enables native speakers to transfer L1 reading skills to L2 reading when reading a foreign language ${ }^{[2]}$ Therefore, can we explain it like this: the pre-structure (The center of gravity is behind.) can best reflect the semantic master-slave relationship because the second unit can meet the normal expectations of readers and should be a continuous relationship; the post-structure (front 
center of gravity) should be a discontinuous relationship, because the conclusion is before the readers need to do further analysis, and the original "normal expectation" has changed, making the reader "a little surprised". The thinking habits of the Han nationality and the British nationality are different, which are manifested in the language that the Han nationality is accustomed to the comprehensive type with the center of gravity behind the sentence, and the sentence pattern is "flowing water type", which forms a logical continuity relationship; English is mainly analytical, the word order is flexible, and the sentence structure focuses on the front, showing a "branch type" sentence pattern, therefore, it forms a logically discontinuous relationship. ${ }^{[10]}$

Fahnestock's research found that the turning relationship is the most difficult to understand (It takes the longest time.), and followed by the causal relationship, the illustrative relationship is the easiest to understand. ${ }^{[8]}$ So for L2 readers whose mother tongue is Chinese, will the preposition and postposition of conditional adverbial clauses have an impact on reading speed and understanding? Based on the above analysis, this paper intends to analyze through two experiments.

\section{Research methods and techniques}

Based on the E-Prime experiment generation system, reading time method and probe technique are used to implement the research. Reading time method is to measure the time for readers to process target information during reading, that is, to measure the time for readers to stay on the target — response time. Through the specific value of residence time, this paper analyzes the speed of readers' processing of target information, and then analyzes the difficulty of understanding reading target. ${ }^{[11]}$ Probe technique, that is, presenting a probe word to the reader, requires the reader to quickly judge whether the word has appeared in the previously read target information or whether it has appeared in the meaning expressed by the target information. The experiment was conducted in one morning and was divided into two stages, namely, sentence-level investigation and text-level investigation.

\section{Experiment 1: investigate the differences of the subjects' understanding of the two structures at the sentence level}

\subsection{Experimental design}

Twelve Chinese-speaking college students with normal eyesight were randomly divided into two groups. From Zhang Zhenbang's New Grammar Course, 32 experimental sentences, 16 pre-structural sentences and 16 post-structural sentences were selected and randomly compiled into the official reading materials. Two groups of college students tested 16 sentences in each group, with 8 sentences in the pre-structure sentence and 8 sentences in the post-structure sentence. In order to control the participants to form certain judgment strategies, the materials also contained 32 filling sentences of other types, all of which were of the same length. The collected data were statistically analyzed by SPSS.

Examples of materials are as follows.

Pre-structure: If the dam breaks in the field,there will be great loss of life and property.

Post-structure: Great loss of life and property will be made if the dam breaks in the field.

Probe word: anxiety

Pre-structure: Unless they were rich, they couldn't afford to live in such an expensive apartment.

Post-structure: They couldn't pay the huge sums for such an expensive apartment unless they were rich.

Probe word: capability

Examples of filling sentences:

Filling sentence 1: John spoke through a microphone so that he could be heard in every room.

Probe word: absolute necessity

Filling sentence 2: He had been prevented by illness from studying, but he passed the examination.

Probe word: earnest attempt

\subsection{Experimental process}

In order to familiarize the subjects with the experimental procedure, a 5-minute pre-experiment was provided before the formal experiment, and the procedure evolution was the same as that of the formal experiment. The formal experiment begins: First, the guiding language appears, and after the guiding language fades out slowly, a "beep" appears to remind that the experiment is about to start. A sentence appears slowly in the middle of the computer screen. The subjects read at their own speed, and the index fingers of the subjects' left and right hands are placed on the D and K keys of the keyboard respectively. The subjects read the sentence and do the corresponding fingertip movements at their own speed. After 
reading a sentence, press the space key to present the next content, and a gaze point lasting 500ms will appear. After the gaze point disappears, a probe word will appear. The subjects are required to quickly and accurately judge whether the word or meaning has appeared in the sentence just presented or is consistent with the sentence meaning. If it has appeared or is consistent, press the D key. If not, press the K key. When "+" appears, it indicates that the next sentence appears. Similarly, in order to avoid the strategic processing caused by repeated presentation of sentences, when programming, set the Experiment List property to Random. The time and reaction of reading target information are automatically recorded by the system with its operation, and the experiment lasts about 10 minutes. Filling materials and pre-experiment data are not included in the statistics.

\subsection{Results and analysis}

See Table 1 for the reading time of the pre-structural sentences and post-structural sentences and the response time of the subjects to the probe words.

Table 1. Average and standard deviation of reading time $(\mathrm{ms})$ and response time $(\mathrm{n}=12)$

\begin{tabular}{ccc}
\hline Sentence structure & Reading time & Response time \\
\hline Pre-structure & $1046 \pm 278$ & $741 \pm 116$ \\
Post-structure & $1250 \pm 312$ & $786 \pm 102$ \\
t & -5.274 & -3.625 \\
P & $<.01$ & $<.01$ \\
\hline
\end{tabular}

T-test of the same group of samples was carried out on the data in Table 1. The results show that the reading time of the subjects is significantly longer in the post-structure than in the pre-structure, with absolute value $t=5.274>t(11,0.01)$ $3.106, \mathrm{p}<.01$. The results show that at the sentence level, there is a very significant difference between the average number of post-structured sentences and the average number of pre-structured sentences, which shows that reading post-structured sentences is more time-consuming and more difficult to understand than reading pre-structured sentences. There is also a significant difference in the response time to the probe words under the two structural conditions, with the absolute value $\mathrm{t}=3.625>\mathrm{t}(11,0.01) 3.106, \mathrm{p}<.01$, which supports the difference in reading time.

\section{Experiment 2: investigate the differences of the subjects' understanding of the two structures at the text level}

\subsection{Experimental design}

The subjects of text reading experiment and sentence reading experiment are the same group of college students. We still select the test materials from Zhang Zhenbang's New Grammar Course, and select eight reading paragraphs. The probe word is one of the words in the background materials in the paragraphs, or the meaning word that has not appeared before, and all the paragraphs have the same length. Similarly, in order to control the subjects to form certain judgment strategies, eight filling segments are provided, and the length and difficulty of filling segments are equal to those of formal segments. The collected data were statistically analyzed by SPSS.

Examples of background materials are as follows.

In the last twenty years or so, some undeveloped countries have increased their food production. Their population, however, have at the same time grown faster, and so their standard of living hasn't improved. What's more, their increase in food production has been achieved at the expense of using up marginal land.

Pre-structure: If they go on increasing their food production, there will be no gain in the productivity of land labour.

Post structure: They will take a risk of losing the productivity of land labour if they go on increasing their food production.

Probe word: standard of living

Example of filling material

Background material:

True, in the woods Wolf was as brave as an honorable dog should be; but what dog is ever brave enough to stand firm against the terrors of a woman's tongue? As soon as Wolf entered the house, his head bent low, his tail lay on the ground or curled between his legs. He went around the house with a guilty look, watching Dame Van Winkle out of the corner of his 
eyes.

Filling sentence 1: Dame Van Winkle was at the slightest sign of her displeasure, and he was ready at any time to run from the room.

Filling sentence 2: He went around the house and was ready at any time to run from the room, for Dame Van Winkle looked a little unhappy.

Probe word: frightenedness

Eight formal reading materials are divided into two parts according to Latin square, and eight filling materials are randomly divided into two parts, which are compiled into formal materials respectively. The experimental operation process is the same as above.

\subsection{Results and analysis}

See Table 2 for the reading time and reaction time of the subjects to the two structures at the text level.

Table 2. Mean time (ms) and standard difference $(\mathrm{n}=12)$

\begin{tabular}{cc}
\hline Sentence structure & Response time \\
\hline Pre-structure & $1178 \pm 356$ \\
Post-structure & $1284 \pm 336$ \\
$\mathrm{t}$ & -3.451 \\
$\mathrm{P}$ & $<.01$ \\
\hline
\end{tabular}

T-test of the same group of samples was carried out for the response time of the probe words at the two structural levels in Table 2. The results show that there are significant differences in the response time of the probe words under the two structural conditions, with absolute value $t=3.451>t(11,0.01) 3.106, p<.01$. The results show that, at the text level, the response time of post-structural sentences to probe words is significantly longer than that of pre-structural sentences. It is clear that post-structural sentences are more difficult to characterize and understand than pre-structural sentences at the text level.

\section{Discussion and conclusion}

The experimental results show that it is more difficult for the clause postposition structure of conditional adverbial clause to form representation than the preposition structure. Since the same research has not been reported up to now, this result should be innovative, but it is basically consistent with the previous research conclusions. ${ }^{[3,8,12]}$ According to the relevant theoretical framework and its analysis of the results, in the prepositional structure sentences in this study, the inference or conclusion that the reader finally gets from the main sentence is based on the prepositional clause (condition), which is a "flowing water" sentence pattern. The front information and the back information are naturally coherent, and the new information is consistent with the original information structure, and only needs to develop continuously in the original structure, which is in line with the thinking mode of the Han nationality that "the focus of the structure is behind". ${ }^{[10]}$ Therefore, the reading time is relatively short, and the response time to the probe words is also short. In the post-structure sentence, the semantic center of gravity of the sentence comes first, and the information behind it belongs to analytical type, which can be "from one to list multiple", making readers spend more time processing the reading information. Therefore, it takes longer time to construct the new structure, and the response time to the probe words is also longer.

This result also verifies Fahnestok's view (1983) to a certain extent, that is, "to meet normal expectations". Therefore, continuity makes it easier to construct representation, whereas discontinuity makes it more difficult to construct representation. In Experiment 2, the background materials under the conditions of pre-structure and post-structure are consistent, and probe words are words that have appeared in the background materials or meaning words that have not appeared. After reading the background materials and corresponding pre-structure sentences and post-structure sentences, the subjects' response to probe words of background materials is consistent with the reading test results at the sentence level, which shows that the post-structure is more difficult to characterize than the pre-structure in both independent sentences and reading paragraphs with background materials, which affects the reading speed and understanding accuracy of the subjects.

Based on the theory of structural construction, this study probes into the differences in the understanding of prepositional clauses and poststructural clauses of conditional adverbial clauses in English reading of Chinese-speaking 
college students from the sentence level and the paragraph level, and analyzes the significance of the differences. As the research is limited to conditional adverbial clauses, whether this conclusion is applicable to other adverbial clauses needs to be further verified. In addition, the sample size of the research needs to be further expanded on the basis of this research. In a word, applying the theories and findings of cognitive psychology to study second language reading is helpful to understand the essence of second language reading, improve second language reading teaching, and improve the level and effectiveness of reading teaching.

\section{Acknowledgments}

In the writing of this paper, the relevant works and journals of many experts and scholars at home and abroad have been referenced, which have been listed in the reference. I would like to pay tribute to the original author. The research work has also received the full support and care of the project team members, and I would like to express my deep gratitude.

This paper is supported by the first-class undergraduate major construction site of Taizhou University (Project Number: 19YLZYB03).

\section{References}

[1] Wang Suiping, Mo lei. Current research progress of text reading. Exploration of Psychology. 2001; 21(3): 20-25.

[2] Wu Shiyu, Wang Tongshun. A study on the transfer of foreign language reading skills under the framework of structural construction. Foreign Language Teaching and Research. 2006; 38(2): 122.

[3] Elizabeth J. The comprehension of logical relations in expositor texts by students who study through the medium of ESL. System. 2006; 34: 432-450.

[4] Wu Shiyu, Hu Wenjie. The role of Chinese in college English learners' reading. Foreign Language Teaching Abroad. 2007; (4): 1-8.

[5] Kintsch W. The role of knowledge in discourse comprehension: A construction-integration model. Psychological Review. 1988; 95: 163-182.

[6] Gernsbacher MA. Language comprehension as structure building. Hillsdale, NJ: Lawrence Erlbaum Associates Inc.; 1990.

[7] Winter E O. A look at the role of certain words in information structure. In: Jones, K P. Harsnell, V. Informatics 3. Proceedings of a conference held by the Aslib Co-Ordinate Indexing Group. Aslib, London; 1975.

[8] Fahnestock J. Semantic and lexical coherence. College Composition and Communication. 1983; (34): 400-416.

[9] Zhang Zhenbang. New English Grammar Course. Shanghai: Shanghai Foreign Language Education Press; 2003.

[10] Liu Hong. Chinese-English language differences and Chinese-English translation. Journal of Southwest University for Nationalities (Philosophy and Social Sciences Edition). 2002; 23(12): 322-324.

[11] He Huizhong, He Lizhong. A survey of discourse reading research methods. Science of Psychology. 2005; 28(6): 1427-1429.

[12] Wu Nini, He Xianyou et al. An experimental study on differences in understanding causality and turning relationship in Chinese reading. Psychological Development and Education. 2009; (2): 73-77. 